\title{
Undergraduate Nursing Students' Perceptions of Obstetric Skills Following High-Fidelity Simulation Experience
}

\author{
Medine Ardic, MSN ${ }^{1}$, GulPinar, Assoc. Prof ${ }^{2}$, Elizabeth Barker, Prof ${ }^{3}$ \\ ${ }^{1}$ Yıldırım Beyazıt UniversityFaculty of HealthScienceNursingDepartment, Ankara, Turkey \\ Cankırı Street, No:3 Ulus Ankara, TURKEY \\ ${ }^{2}$ NursingDepartment, YildirimBeyazitUniversityFaculty of HealthSciences, Ankara, Turkey \\ Cankırı Street, No:3 Ulus Ankara, TURKEY \\ ${ }^{3}$ Director of Office World HealthOutreach,The Ohio StateUniversity, The Ohio \\ StateUniversityCollege of Nursing, Newton Hall, 1585 NeilAvenue, Colombus, OH 43210 \\ ${ }^{1}$ ardicm@yahoo.com, ${ }^{2}$ gpinar_1@hotmail.com, ${ }^{3}$ barker.203@osu.edu
}

\begin{abstract}
:
Aim: An evaluation of the perceptions and opinions of nursing students on the effect of high-fidelity simulation model on the development of obstetric skills was examined in this study.

Method: There search was conducted on the third year students in the United States of America at the College of Nursing at The Ohio StateUniversity in 2014 using adescriptive research design. T test was used in the statistical analyses for the average, standard deviation, percental distributions, and independent samples. Results: Nearly the entirety of the students in there search reported that they found thesimulation model significant for gaining obstetric skills, and their communication skills with the patients and team improved.The debriefing sessions were identified as important to the observance of the effectiveness of the initiatives and that the simulation model reflected the real clinicsetting in application. Furthermore, $85.7 \%$ of the students were satisified with performing application with the simulation model and 95.2\% suggested this model for developing obstetric skills.

Conclusion:The simulation favorably affected the learningexperiences of students. The students reported that this model was quite effective on self-afficacy, professional skills, self-confidence, problem solving, communication, and teamwork.Use and generalization of the simulation application as an education method in nursing education is suggested since it contributes to students.
\end{abstract}

Keywords: High Fidelity Simulation, Simulator, Nursing Students, Obstetric skills

\section{INTRODUCTION}

Simulation is an active teaching techniquein healthcare education as it mimics a real-life event [1]. Simulation provides unique oppurtunities for nursing studentstodeveloptechnical clinical skills, an holistic approach, improved communication skills, appropriate decision-makingandimproved critical thinking using appropriately designed scenarios $[2,3]$. Students may not experience these situations in actual clinical settings due to low frequency of complex patient presentations and limited time in the maternity clinical setting during the undergraduate clinical program. Another advantage to simulation is that it permits interprofessionaleducation without placing actual patients at risk in the real clinical setting [4]. The World Health Organization (WHO) has provided standards for healthcare education to increase understanding professions and recommended the use of simulation for safe patient care in collaborative teams [5]. There are many different types of simulation such as mannequin simulator, partial task simulator, standardized patients, hybrid simulations, virtual simulations, in-Situ simulations. Simulation is not a new technique and many students or professionals have widely used simulation for years across all disciplines [2]. However, technologically high fidelity simulation (HFS) is a relatively new educational tool. HFS refers to simulation scenarios that closely replicate the true environment, making it easier for participants to believe in the reality of the situation depicted, and thus to apply realistic responses $[6,7]$. 
Human error and communication problems are crucial elements to avoid in nursing education. Nowadays, nursing students have a number of challengesas they learn to improve their competency. There are a reduced number of clinical settings where students may participate in direct nursing care to the patients. Consequently, the use of simulation in modern nursing education has developed significantly within the past decade $[2,8,9]$. Simulation is also particularly useful for enabling students to evaluate their own needs.Many studiesthat analyzed the quality of health care supported the demand for higher quality healthcare [10-13]. Faulty in many educational institutions believedthat the development of critical thinking skills isneeded to work in the increasingly complex clinical environment.Simulation is an effective way to achieve this goal. Advanced simulationhas been viewed as a method that may be integrated into all levels of curricula in healthcare content [8]. Despite the increased use of simulation and the attention received [3], the integration of simulation into nursing curricula has been inconsistent. Unfortunately, evidence of effectiveness of simulation traininghas not been systematically evaluated, especially in nursing education. For this reason, theaim of this research was to investigate nursing students' perception of simulation effectiveness in teaching obstetrics skills.

Research questionsthat were developed for this study are :

1) How satisfied are the nursing students with the simulation experience?

2) What is the nursing student's self-report of simulation effectiveness?

\section{METHODS}

In this study, the participants enrolled were third year nursing students in 2014 at a large land grant university in the Midwest of the United State. The students have completed a theoretical course in obstetric nursing covering prenatal care, delivery and postnatal care as third semester junior in obstetric nursing. Adescriptive, correlational design was used for this study.Participants were 80 undergraduate junior nursing students in 2013. All students participated in the maternity educational program.

The inclusion criteria for the students in the current study were (a) that the students did not attend any other course regarding obstetric nursing skills at another university (b) that he or she agreed to participate and (c) that students enrolled in the gynaecology and obstetric nursing course. The exclusion criteria were 1) Nursing students who were under the age 19, and 2)thosewho did not volunteer to be in the study.Design and ethical approval was obtained from the University Institutional Review Board prior to commencing this study.Pariticpation in the study was voluntary and students provided written consent prior to engaging in the study.

Two instruments were employed for data gathering.

1) The High Fidelity SimulationEvaluation Form; an 18 item schedule that scores"yes" or "no"questionsusing descriptive behavioral anchors to define team performance including demographic data such as gender, age, and role played during simulation activity.This form also includes a 7-item scale to measure students' perception of the obstetric simulation experiences. Responses were rated on a 5-point Likert scale with values ranging from 1 (strongly disagree) to 5 (strongly agree).

2) Semi-structured Interview Form; there were 12 open-ended questions related to the things they have learned, things students wished were focused on, and their preference of having a high fidelity mannequins in the simulations. The time needed to complete the questionnaire was about 20 minutes.

Independent sample t-test were used to examine team performance using SPSS 19.0 (Inc. Chicago, Illinois). In statisticalanalysis number, percentage, mean and standard deviation were used. Tests to analyze the relation between the dependent variables and independent variables were student $t$ test. Significance level was defined as $\mathrm{p}<0.05$ in this study.

\section{Application}

Phase-1-Facilities; the simulation lab utilized the Noelle-Gaumard high fidelity simulator and a human embedded patient. Faculty, staff or volunteers served as the patient, family members or health care professionals as called for in the case scenarios. Student participants were in the simulation lab set up for each scenario. Classroom observers were sitting in a classroom watching the simulation unfold via a web-based system, which also recorded the scenario. 
Phase-2-Scenario and debriefing; three different obstetrical simulation session scheduled on thesame day were the basis for the study. Simulations were divided into three separate three-hour sessions with different scenarios in each session.First session case scenarios pertained to (1) preeclampsia/hyperemesis, (2) teen pregnancy/preterm labor, and (3) shoulder dystocia/postpartum hemorrhage. All scenario teams (students of 5) were orientated to the manikin and environment prior to engaging in the simulation experience. Students were randomly assigned to teams of three for each patient case scenario $(n=15)$. The simulation experience consisted of 15 minutes scenarios each followed by 20 minutes of structured debriefing. All simulation experiences were recorded continuously using wide-angle video cameras from different angles. Debriefing followed a standart interview method including a reaction phase, an exploration phase. All observers involved obstetric scenarios. Simulation objectives were discussed as a team performance. The purpose of the debriefing session was to discuss leadership, roles and responsibilities, communication, awareness and skills of obstetric proseduresthat occurred during the scenario. The study was conducted in the simulation lab and classroom. All clinical case scenarios for simulation were designed to be similar to what could be encountered with a typical patient in a hospital setting.

Phase-3-evaluation; at the end of the simulation session, the students completed the questionnaire, which focused on their perceptions of simulation effectiveness. While 80 students were enrolled in the course, participation in the study was voluntary. The questionnaires took about 15-20 minutes to be completed by students.

\section{ReSUlts}

The age average of the students included in the research sample was 21.09 \pm 2.62 (min:19; max:32).81\% of the students were female $(n=16)$, and $19 \%$ were male $(n=5)$. The thoughts of the students about their simulation experiences were examined in the study. Accordingly, $95.2 \%$ of the students expressed that they noticed the importance of HFS model in a learning setting and HFS model facilitated learning, $97.3 \%$ indicated that it contributed to the development of their nursing skills, $90.5 \%$ reported that the debriefing application ensured observance of the effectiveness of the initiatives, and in addition, $33.3 \%$ of the students reported that HFS model caused them to feel stress.

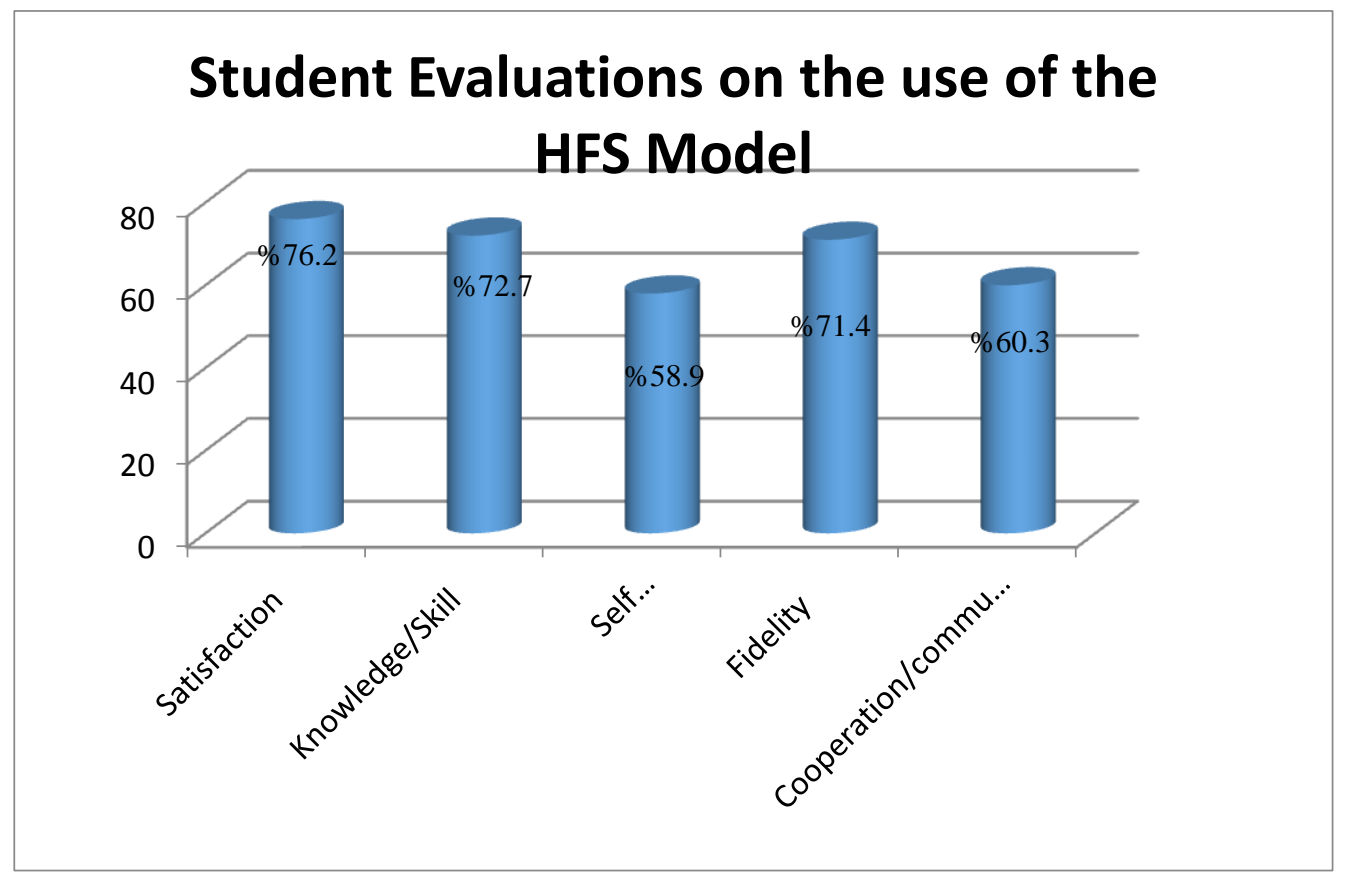

Figure1. Student evaluations on the use of HFS model

In Figure 1, the students inidcated that they were satisfied with the simulation applications in general (76.2\%), they gained knowledge/skills (72.7\%), developed self-confidence/critical thinking skills (58.9\%), they performed clinical application close to reality $(71.4 \%)$, and they improved their cooperation and communication skills $(60.3 \%)$. According to these results, it was determined that the students had high level of positive feedback about the study with HFS model. 
Table1. The distribution of evaluations and suggestions of the students about HFS model

\begin{tabular}{|l|l|l|}
\hline Evaluation & n & $\%$ \\
\hline Simulation duration is appropriate. & 12 & 60.0 \\
\hline Applications with simulated patients are more realistic. & 18 & 90.0 \\
\hline I had an opportunitytoobserve in-teamroles. & 12 & 60.0 \\
\hline I think that its cost is very high. & 10 & 50.0 \\
\hline Suggestion & & \\
\hline The application setting must be more realistic. & 3 & 15.0 \\
\hline Active participation of all students in the application must be ensured. & 5 & 25.0 \\
\hline More information must be provided about the scenario. & 4 & 20.0 \\
\hline Different disciplines must be integrated into the scenario. & 8 & 40.0 \\
\hline Scenario number must be increased. & 6 & 30.0 \\
\hline
\end{tabular}

In Table 1, 90\% of the students stated that the simulated patient (live actor) was more realistic in comparison to the human simulator, and $60 \%$ reported that they had an opportunity to observe the roles within the team and that the simulation duration was adequate. Moreover, the students had suggestions to integrate different disciplines in the scenario (40\%), to apply a higher number of scenarios, and to ensure active participation of all students $(30 \%)$.

InTable 2 , it is shown that the total score average of the students about the effectiveness of HFS model is 54.62 \pm 8.46 . The students expressed that HFS was an effective model (8.00 \pm 1.14$)$, contributed to learning $(8.28 \pm 1.27)$, and encouraged them to learn $(8.09 \pm 1.34)$, ensured adequacy in clinic application(7.57 \pm 1.72$)$, and they felt successful in clinical applications $(7.90 \pm 1.89)$, they felt good during simulation (7.43 \pm 1.60$)$, and it prepared them for real clinic setting $(7.33 \pm 1.53)$.

Table 2. The distribution of the evaluations of the students about the effectiveness of HFS model

\begin{tabular}{|c|c|c|c|c|}
\hline HFS Model Evaluation & Min & Max & Mean & SD \\
\hline 1. It is an effective education model. & 5.00 & 10.00 & 8.00 & 1.14 \\
\hline 2.It contributed to my learning. & 5.00 & 10.00 & 8.28 & 1.27 \\
\hline 3. It encouraged learning. & 5.00 & 10.00 & 8.09 & 1.34 \\
\hline 4. It ensured my competency in clinical application. & 3.00 & 10.00 & 7.57 & 1.72 \\
\hline 5. I found myself to be successful during simulation. & 4.00 & 10.00 & 7.90 & 1.89 \\
\hline 6.I felt good during simulation. & 4.00 & 10.00 & 7.43 & 1.60 \\
\hline 7. It enabled me to prepare myself for a real clinical setting. & 5.00 & 10.00 & 7.33 & 1.52 \\
\hline Total & 31 & 70 & 54.62 & 10.48 \\
\hline
\end{tabular}

Table3. The distribution of the averages of HFS model effectiveness scores according to personal characteristics

\begin{tabular}{|l|l|l|l|l|l|}
\hline Individual Features & n & Mean \pm SD & Min & Max & Analysis \\
\hline Age & & & & & \\
\hline $19-20$ & 11 & $55.81 \pm 10.41$ & 35 & 70 & $\mathrm{t}=0.672$ \\
$>20$ & 9 & $53.30 \pm 5.88$ & 43 & 63 & $\mathrm{p}=0.071$ \\
\hline Gender & & & & & \\
\hline Female & 16 & $56.05 \pm 7.11$ & 43 & 70 & $\mathrm{t}=1.680$ \\
Male & 5 & $48.50 \pm 12.06$ & 35 & 61 & $\mathrm{p}=0.0259$ \\
\hline
\end{tabular}

Table 3 shows that female students $(56.05 \pm 7.11)$ found HFS model more effective than male students did $(48.50 \pm 12.06)(\mathrm{p}<0.05)$. Nevertheless, there is no statistically significant difference between the ages and the averages of HFS model effectiveness scores $(\mathrm{p}>0.05)$.

\section{DisCuSSION}

The use of simulation and integrated curricula in nursing education provide an experience based learning opportunity for the students, an increase in the student' s knowledge and self-confidence, and supports the development of clinical decision-making skills [12]. In a study with a total of 151 medical and nursing student participants, positive attitudes of the simulation model have been reported on learning by Ker et al. reported [14]. In Southeastern University, Bambini et al. evaluated the efficacy of HFS model related to postpartum examination on 112 nursing students during their initial clinical course in a prelicensure program. They detected a significant increase in overall selfefficacy of the students [15]. The students participated in the present study expressed that HFS is an effective and appropriate training methods. The mean rating score with 54.62 \pm 10.48 ( $\min : 7, \max : 70)$, 
which is higher than those in the general average score, revealed that the students accepted the model was effective. The results of this study were consistent with the literature.

The physical environment has a significant contribution on learning.In this sense, the educational environment must be prepared beforehand in accordance with the learning objectives and the students must be oriented in order to be successful [3]. The majority of students $(71.4 \%)$ in this study think that the physical environment is important. In addition, the students think that simulated patient practices reflect the reality of the physical environment more.

In the studies carried out by Gordon et al. and Brannan et al., the majority of the both students and educators rated that HFS model ensures an excellent educational environment $[16,17]$. In a study by Smith ve Roehrs, HFS were significantly correlated with a significant effect of simulation on knowledge of advancedcardiovascular life support [18].Ackermann (2009) examined the effect of simulation on the acquisition of cardiopulmonary resuscitation knowledge. A quasi-experimental study showed that simulation had a statistically significant effect on knowledge retention of cardiac care among 69 junior-level nursing students in the Northeastern United States [8]. Kaplan and Ura (2010) used simulation to enhanceclinical performance self-confidence and safely care for numerous patients in senior nursing students ( $\mathrm{n}=97)$ [19]. Feingold, Calaluce, and Kallen(2004) conducted a study to examine nursing student $(\mathrm{n}=65)$ perceptions about high fidelity simulator using a 20 -item tool.In that study, findings showed that while the majority of students agreed the simulations were a realpracticeand valuable[20]. Schoening, Sittner, and Todd (2006) reported the importance of simulation as an effective means of providing a realistic and practical environment [21]. In a recent studyby Robertson et al 2009, no statistically significant improvement in knowledge on obstetric crisis was found, but there was a positive change in team attitude and team performance [22]. Contrarily, a randomised controlled trial reported change in knowledge and skills after training emergency obstetric including eclampsia, shoulder dystocia, breech delivery, and postpartum haemorrhage $(\mathrm{p}<0.001)$ [23].

The authors believe that one of the main challengesof nursing education is to provide opportunities for the students toeffectively transfer theoretical knowledge into clinical practice.In the acquisition of nursing skills, clinical experience based on simulation is an important component of professional education [1]. Clinical experience aims to develop the student's critical thinking, analysis, psychomotor, comunication and management skills, as well as to help for gainingconfidenceduring their performingthe nursing profession. In an ideal environment, clinical training provides learning experiences, which develop professional nursing skills. Clinical experiences can also be a significant source of stress and anxiety for students. This may have a deleterious effect on their learning $[1,3]$. Hovewer, in our study, $95.2 \%$ ofthe students expressed that the simulation applications facilitate learning. In the sample population, 33\% stated that the applications also could causeanxiety. In a study on nursing students, it was determined the students to have higher levels of anxiety after thesimulation application [4]. As consistent with the literature, our study supportsthat the simulation training method creates stress on students, reduce this stressplanning before scenarios,and giving enough information to student's thoroughcomprehensive briefings is of great importance. Additionally, obtaining student opinions and evaluations on the use of the simulation modelfor skills training and orientation of students about the scenario before the simulationcan be useful. In this study, we observed that the students expect to be informed more about the simulation scenario. Prior to the simulation, faculty would be well advised to determine if the scenarios are appropriate to the student's level, course material and objectives.Therevisionsmay increase student satisfactionby reducing stress levelsand increasing student engagement.

In a study about the effectiveness of the HFS model on the update of emergency obstetric skills, it was found that the training has a positive contribution on emergency obstetric skills intechniques, and behavioral and cognitiveskills [24].Theintegration of this method into the other courses may be very useful.In a study carried out with nursing students, Pınar ve Doğanreported that the majority of students also suggested theintegration of HFS model into the other courses since simulation models reduced learning time, developed a sense of confidence and motivation, and showed what they can do as a nursing student [4]. DeVita et al.,in their study of 138 nursing students, detected that the students develop their nursing skills related tocrisis management, intensive care nursing practice, the application of aspiration and life support [25]. Guhde determined that nursing education performed by 
HFS model increased their satisfaction leveland developed critical thinking skills of undergraduate students ( $\mathrm{n}=134)$ in Ohio [26]. Childs and Sepples conducted a study using complex patient care scenarios in simulation practice. In the study, it was notified the studentshave found that learning opportunities are versatile and rich through simulation practice application, and thereforethey expressed simulation practice should be taken place in the curriculum [27]. Our findings are similar toresults in theother reports in the literature, and supports the finding that simulation improvesthe clinical skills.

\section{Conclusion}

This study supported the concept that simulationis a satisfactory learning experience for the nursing students. It also confirmed that this technique might be, in the students' opinion, an effective method for learning clinical skills. There are several indications for further investigation. First, a study with a different design that compares the experiences of students in different institutions may be helpful. Second, there should be a study conducted with a larger study population, perhaps comparing students who are at different levels of study than just the third or junior year. This would help to determine if simulation is appropriate at different levels of educational preparation. Third, an interdisciplinary investigation involving several different health care disciplines might shed light on the efficacy of this technique on a more global basis. Fourth, the confounding concept of anxiety produced by the technique and an evaluation of its effects on the quality of learning might also be an important area of investigation.

\section{ACKNOWLEDGEMENT}

The authors would like to acknowledge the contribution of the faculty staff and nursing students of The Ohio State University.

\section{DISCLOSURE}

We certify that no actual or potential conflicts of interest in relation to this article exist.

\section{Authors' CONTRIButions}

Ardic M, Pınar G. and Barker E: Study development, Data Collection and Manuscript writing

\section{REFERENCES}

Katoue, M. G., Iblagh, N., Somerville, S., \& Ker, J. (2015). Introducing simulation-based education to healthcare professionals: exploring the challenge of integrating theory into educational practice. Scottish Medical Journal,60(4), 176-181.

Jeffries, p. (2005). A framework for designing, implementing and evaluating simulations used as teaching strategies in nursing. Nurse education perspectives, 26(2), 96-103.

Berragan L. (2014).Learning nursingthroughsimulation: A casestudyapproachtowards an expansive model of learning, NurseEducationToday, 34,1143-1148.

Pinar, G., Knight, C. C., Gaio, V. P, Watts, P. I., Dailey, K. D., Britt, S. E., Catron, K, S., \&Zengul, F, D. (2015). TheEffects of highfidelitysimulation on nursingstudents' perceptionsand selfefficacy of obstetricskills, International Archives of NursingandHealthCare, 1(1), 1-7.

World Health organization (WHO) (2010). Framework for Action on Inter professionals Education and Collaborative Practice. Genevo: Author. http://whqlibdoc.who.,nt/hq/2010/ WHO_ HRH_HPN_10.3_eng.odf

Tawalbeh, L. I., \&Tubaishat, A., (2014). Effect of simulation on knowledge of advanced cardiac life support, knowledge retention, and confidence of nursing students in Jordan. Journal of Nursing Education, 53, 38-44.

Kim, Y.H., \& Jang, K.S. (2011).Effect of a simulation-based education on cardio-pulmonary emergency care knowledge, clinical performance ability and problem solving process in new nurses. Journal of Korean Academy of Nursing, 41, 245-255. doi:10.4040/jkan.2011.41.2.245.

Ackermann, A.D. (2009). Investigation of learning outcomes for the ac- quisition and retention of CPR knowledge and skills learned with the use of high-fidelity simulation. Clinical Simulation in Nursing, 5, 213-222. 
Hoffmann, R., O’Donnell, J.M., \& Kim, Y. (2007).The effects of human patient simulators on basic knowledge in critical care nursing with undergraduate senior baccalaureate nursing students.Simulation in Healthcare, 2, 110-114.

Adamson, K. 2010. Integrating human patient simulation into associate degree nursing curricula faculty experiences, barriers, and facilitators. Clin.Simul.Nurs.6,e75e81. http://dx.doi.org/ 10.1016/j.ecsns.2009.06.002.

Akhtar-Danesh, N., Baxter, P., Valaitis, R.K., Stanyon, W., Sproul, S., 2009. Nurse faculty perceptions of simulation use in nursing education.West. J. Nurs. Res. 31 (3), 312-329. http://dx.doi.org/10.1177/0193945908328264.

Howard, V.M., Englert, N., Kameg, K., Perozzi, K., 2011. Integration of simulation across the undergraduate curriculum: students and faculty perspective. Clin. Simul.Nurs.7 (1), e1-e10.

Sears, K., Goldsworthy, S., Goodman, W., 2010. The relationship between simulation innursing education and medication safety. J. Nurs. Educ. 49 (1), 52-55.

Ker J, Mole L, Bradley P (2003). Early introduction to inter professional learning; a simulated wardenvironment. Medical Education, 37, 248-255.

Bambini D, Washburn J, Perkins R.(2009). Outcomes of clinical simulation fornovicenursingstudents: communication, confidence, clinicaljudgment.NursEducPerspect, 0(2):79-82.

Gordon J, Wilkerson WM, Shaffer DW, Armstrong, E. (2001). Practicing Medicin without Risk: Students' and Educators' Responsesto High-fidelity Patient Simulation. Academic Medicine 76(5):469-472.

Brannan JD, White A, Bezanson JL. (2008). Simulator effects on cognitive skills and confidence levels. The Journal of Nursing Education, 47(11):495-500.

Smith, S., \& Roehrs, C. (2009). High-fidelity simulation: Factors correlated with nursing student satisfaction and self-confidence. NursEducPerspect, 30(2), 74-78.

Kaplan, B., \&Ura, D. (2010). Use of multiple patient simulators to enhance prioritising and delegating skills for senior nursing students. Journal of nursing education, 49(7), 371-377.

Feingold CE, Calaluce M, Kallen MA. Computerized patient model and simulated clinical experiences: Evaluation with baccalaureate nursing students. J Nurs Educ. 2004; 43(4):156-63

Schoening, A.M., Sittner, B.J., \& Todd, M.J. (2006). Simulated clinical ex- perience: Nursing students' perceptions and the educators' role. NurseEducator, 31, 253-258.

Robertson B, Schumacher L, Gosman G, Kanfer R, Kelley M, DeVita M. Simulation-based crisis team training for multidisciplinary obstetric providers. SimulHealthc 2009; 4:77-83.

Crofts JF, Ellis D, Draycott TJ, Winter C, Hunt LP, Akande VA. Change in knowledge of midwives and obstetricians following obstetric emergency training: a randomised controlled trial of local hospital, simulation centre and teamwork training. BJOG 2007; 114: 1534-41.

Walker D., Cohen S., Fritz J. et al. Team training in obstetric and neonatal emergencies using highly realistic simulation in Mexico: impact on process indicators. BMC PregnancyandChildbirth, 2014, 14:367.doi: 10.1186/s12884-014-0367-1

DeVita, MA., Schaefer, JL.,Wang, H. \&Dongilli, T. (2005). Improving medical emergency team (MET) performance using a novel curriculum and a computerized human patient simulator. Quality and Safety in Health Care, 14, 326-331.

Guhde, J. (2011).Nursing studentse perceptions of the effect on critical thinking, assessment and learner satisfaction in simple versus complex high-fidelity simulation scenarios.Journal of nursing education, 50(2), 73-78.

ChildsJC, Sepples S (2006).Clinical teaching by simulation: Lessons learned from a complex patient care scenario. Nursing Education Perspectives 27, 154-158. 